\title{
Lessons Learnt and Pathways forward for National Civil Registration and Vital Statistics Systems after the COVID-19 Pandemic
}

\author{
Matthew Kelly ${ }^{1, *},\left(\mathbb{D}\right.$, Gloria Mathenge ${ }^{2,(\mathbb{D})}$, Chalapati Rao ${ }^{1,(\mathbb{D})}$ \\ ${ }^{1}$ Department of Global Health, Research School of Population Health, College of Health and Medicine, Australian National University, \\ 62 Mills Road, Canberra, ACT 2600, Australia \\ ${ }^{2}$ Statistics for Development Division, Pacific Community (SPC), Noumea, New Caledonia
}

\section{ARTICLE INFO}

Article History

Received 25 February 2021

Accepted 02 April 2021

Keywords

Mortality

vital statistics

COVID-19

\begin{abstract}
The COVID-19 pandemic has had a substantial impact on government services in many areas, including Civil Registration and Vital Statistics (CRVS). However, the pandemic has also highlighted the importance of recording of mortality and causes of death, with some potentially positive impacts for longer term CRVS strengthening, including: (1) increasing online provision of registration services (2) reporting of mortality statistics from settings which had not previously done so (3) improved intersectoral cooperation, particularly with the health sector, improving the ability to record deaths and (4) increased awareness among governments and public of the importance of mortality statistics. Now, it is pressing for national governments, and international organizations working to strengthen CRVS systems, to evaluate the effectiveness of strategies adopted over the last year, and use lessons learnt to catalyse broader sustainable CRVS improvement strategies, providing governments with essential data on mortality and causes of death into the future.
\end{abstract}

(C) 2021 The Authors. Published by Atlantis Press International B.V. This is an open access article distributed under the CC BY-NC 4.0 license (http://creativecommons.org/licenses/by-nc/4.0/).

\section{INTRODUCTION}

Civil registration is defined as the universal, compulsory, continuous, permanent and confidential recording of the occurrence and characteristics of all vital events, including births, deaths and marriages. The main function of the civil registration process is to provide people with the legal documentation required for proving identity and civil status. However, these registered events are also then compiled into population level vital statistics. It is a crucial function of government to ensure that every vital event is recorded and compiled. Taken together, national systems that organise all these functions are termed as national Civil Registration and Vital Statistics (CRVS) systems [1]. Data collected by CRVS systems are utilised in an array of government planning and assessment processes. For the health sector, registration of births, deaths and causes of death are of importance for overall health policy planning and for the assessment of health programmes and services [2].

Before 2020 most low and middle income countries globally were already facing significant challenges to the collection of data on vital events, and particularly mortality and causes of death [3]. The COVID 19 pandemic which has affected most countries has had the potential to significantly affect the operations of governments in all areas including in the collection of information on vital events through civil registration systems, as well as the compilation of this

"Corresponding author. Email: matthew.kelly@anu.edu.au information and the production of vital statistics. The pandemic may therefore also exacerbate problems in settings where CRVS systems were already weak.

At the same time however, international efforts to track and monitor the progress of the COVID-19 pandemic have made clear the importance of CRVS systems as the best source of information on mortality caused directly and indirectly by the disease. The collection of timely and reliable data on the progress of the pandemic are essential to accurately measure the impact as well as to inform epidemic control strategies [4]. This is particularly the case where testing regimes are weak and measuring mortality, including excess mortality, may be the most effective way to trace the progress of the pandemic [5].

In March 2020, the United Nations Legal Identity Task Force (UNLIATF) launched a survey to all countries seeking information on how CRVS systems had been impacted by the pandemic, and various aspects of the national responses to this impact [6]. This survey was completed by 61 countries with nearly half from the African continent. A wide variety of impacts on CRVS operations were reported by responding countries including the complete closure of registration offices in seven countries, and in about half of countries a partial closure or significant changes to operations. These changes include limiting operations to the registration of only deaths in some settings, or only births, limiting office hours and staffing, setting up delayed reporting systems, and ceasing of rural outreach registration services. In many settings resources 
for CRVS operations were reduced due to competing demands from COVID response. In some countries budgetary allocations and human resources for CRVS were diverted to support COVID response, in others development partners diverted resources previously dedicated to CRVS strengthening to support COVID 19 response.

To help alleviate some of these challenges various international bodies have developed guidelines for maintenance of CRVS operations during the pandemic. The UNLIATF, made several recommendations for national governments to make arrangements to introduce hygiene measures, review staffing patterns to minimise direct human contact during CRVS processes, to make provisions for electronic services, and develop inter-sectoral collaboration to share workload and administration responsibilities, among others [6,7]. The United Nations Development Program also issued supplementary guidance regarding the adoption of various forms of electronic and digital services for CRVS operations, with a focus on the various aspects of privacy, confidentiality and security of data [8]. From another perspective the World Health Organization (WHO) issued specific technical guidance for medical certification of causes of death in COVID cases, along with use of specific codes for suspected and confirmed COVID infection [9]. Finally, Vital Strategies, a non-governmental organization that provides technical assistance for CRVS operations in developing countries, issued a technical package for implementation of Rapid Mortality Surveillance programs for monitoring the impact of COVID-19 [10]. This technical package included guidance for notification of mortality events identified through the Rapid Mortality Surveillance program to the nearest CRVS office or local registrar.

Despite the disruptions to CRVS system operations due to COVID-19 described above, governments in many settings have been making changes to official procedures and operations, partly induced by the guidelines from the UN agencies. These changes have the potential to have positive longer term outcomes for the collection of information particularly on mortality. Table 1 below summarises these changes as reported by countries in the UNLIATF survey.

\section{POSITIVE OUTCOMES FOR CRVS SYSTEMS ARISING FROM COVID-19}

\subsection{Operational Changes}

The most notable and potentially beneficial change has been the move toward increasing digitisation of CRVS systems and the provision of online registration services. Around one half of responding countries reported some move toward online services. In some settings such as Djibouti and Sri Lanka a temporary online system was set up for the public to pre-request CRVS services. In other countries already existing online services were being strengthened to cope with increased demand (e.g. Georgia, Rwanda, Tanzania, Tunisia, Ecuador). In Afghanistan, Bangladesh, Sierra Leone and Kenya new digitisation plans are underway. Web based certificate provision is being used in Colombia, and in the Solomon Islands registration forms are available online and can be emailed for processing. Finally, in countries such as Australia, New Zealand and Bahrain fully online services were already available for registration. The benefits of CRVS service digitisation are clear during the COVID-19 pandemic, removing the need for physical contact and preventing disease spread, and potentially providing more timely data. The benefits will go beyond this however. In the longer term digitisation has the potential to improve the collection of CRVS data particularly for remote and hard to access communities, to improve efficiency and streamline services and to improve the ability to share data among government agencies and to compile data for the production of vital statistics. It should be noted that although these online services have the potential to improve the production of data on mortality, the provision of the entire suite of civil registration procedures online will require substantial legislative and procedural change, given the requirement in most settings of personal presentation to validate information prior to receiving the legal documents produced by the registration system.

\subsection{Improved Intersectoral Cooperation}

A second change which has occurred due to the challenges of COVID-19 but which has the potential for longer lasting benefit is the improvements in intersectoral collaboration between government agencies, particularly with the health sector. Around $20 \%$ of country responses to the UNLIATF survey referred to this approach. Registration staff are working more closely with community health workers and health facilities to ensure registration without the family involvement. Data sharing between CRVS and health sectors has also been improved. The involvement of the health sector in registration has been particularly recognised due to the essential nature of health services and their continuity despite any lock down periods. In addition, even births and deaths that occur at home often come to the notice of community health staff, who then have an important role in notification and subsequent registration of these events, according to the prevalent or modified local practices in place. Again here the COVID-19 pandemic responses have potential long term benefits for CRVS systems if these connections can be formalised and maintained.

Table 1 Changes to working procedures and official arrangements and recommendations

Changes to registration procedures or official recommendations

\begin{tabular}{lccccc}
\cline { 3 - 5 } Region & $\begin{array}{c}\text { Number of countries } \\
\text { responding to survey }\end{array}$ & $\begin{array}{c}\text { Digital services } \\
\text { strengthened or } \\
\text { developed }\end{array}$ & $\begin{array}{c}\text { Special instructions } \\
\text { or arrangements for } \\
\text { registration }\end{array}$ & $\begin{array}{c}\text { Public } \\
\text { communication }\end{array}$ & $\begin{array}{c}\text { Improved intersectoral } \\
\text { collaboration }\end{array}$ \\
\hline Africa & 30 & 9 & 9 & 6 & 4 \\
America & 9 & 7 & 5 & 0 & 5 \\
Asia & 14 & 10 & 5 & 2 & 2 \\
Oceania & 8 & 4 & 1 & 1 & 2 \\
\hline
\end{tabular}


This will require amended legal frameworks to allow full data sharing in many settings where registration is operated by other sectors. However, the health sector is well placed to improve the reporting of births and deaths and has a crucial role in reporting of cause of death for vital statistics purposes.

\subsection{Mortality Data Reporting Improvements}

For monitoring the impact of the COVID-19 pandemic there is, as discussed above, a need for timely and accurate mortality statistics. These should include accurate population level data on deaths associated with COVID 19 infection, by age and sex group, on a daily/weekly/monthly basis, to be able to assess the magnitude of the pandemic. It is also desirable that for each death, all the multiple causes listed on the medical certificate of cause of death are made available, for detailed analysis of underlying and associated cause mortality patterns from co-morbid conditions [11]. In addition, there is also the need for accurate data on all deaths occurring in the population for the same periods. Further, a complete assessment of the pandemic would also require a times series of data on deaths in the population of interest across the same months during previous years, to evaluate the 'excess mortality' in the population that could be attributed to the COVID 19 pandemic [5]. Excess mortality, or the difference between deaths observed in specific time periods with the expected number of deaths in the same time period (based on historical mortality patterns) is useful in all settings. It allows the measurement not only of deaths directly caused by the COVID-19 virus, but also for measuring deaths indirectly caused by the pandemic, either through delays in screening and diagnosis, or because of overwhelmed health systems.

The WHO in 2018 produced vital statistics 'data usability' score for countries which report such data to the international body [12]. For a large proportion of the countries who responded to the UNLIATF survey no such score could be calculated as countries are not reporting to the WHO. While this suggests that processes for compilation and submission of vital statistics may not be in place in these countries, a scrutiny of international data sources on COVID-19 deaths indicate that all of the survey respondent countries were actually reporting deaths from COVID-19 to the global COVID-19 mortality reporting facility established by the WHO [13]. This suggests that certain agencies (possibly within the health sector) of these countries were performing the function of data compilation and submission for COVID-19 mortality as part of the global pandemic surveillance program.

\subsection{Increased Awareness of Importance of CRVS and Mortality Reporting}

The new onus on reporting deaths from COVID-19 has potentially provided the impetus to improve mortality recording systems, such that these countries could establish data standards for mortality statistics that would be compliant with criteria for their inclusion in the routine WHO mortality database [12]. This development shows that the COVID-19 pandemic is providing an opportunity for many countries to improve data collection and reporting, and hence the experiences of this pandemic will potentially have longer term impacts on CRVS systems globally.
Evidence is now emerging that the increased attention to the collection of mortality data during the COVID-19 pandemic could lead to more sustainable improvements in CRVS systems. In Africa for example many countries including Chad and Liberia are beginning to implement verbal autopsies as part of routine mortality data collection to improve cause-of-death data quality. However, these mortality data are a health sector initiative and would need to be integrated with CRVS strengthening programs to improve overall mortality data. Also, as mentioned above the increased use of technology in CRVS systems is likely to be sustained. For example in Rwanda and Mozambique smartphone based death reporting and registration will continue after the pandemic [14]. There has also been an increase in public awareness and concern about the need to improve the collection of mortality data reflected in numerous media reports through 2020 [15-17], reflecting the increased government and public awareness of the importance of death reporting.

\section{CONCLUSION}

The COVID-19 pandemic has had a profound impact on almost all countries globally. The rapid spread of the disease led to government mandated shut downs and restrictions to services including for civil registration, with resulting impacts on the ability of countries to compile vital statistics. However, in the longer term the changes caused directly and indirectly by the pandemic may have some positive outcomes for the collection of data on mortality and overall CRVS systems in settings where they have had limited functionality to date. The first of these is simply the increased awareness of the importance of producing data on infections and mortality to track and respond to the pandemic. In countries which have previously not reported data on mortality to the WHO, COVID-19 deaths are being reported and compiled indicating the potential capacity for improvements in overall mortality registration. This situation highlights the second positive outcome from the pandemic in terms of improved intersectoral cooperation in the recording and reporting of deaths. The importance of the health sector in recording mortality, even during national shut downs has highlighted the need for closer data sharing and collaboration between the health sector and the CRVS system, and this is indeed occurring in many settings. Finally, the move toward digitisation of registration services has been accelerated during the pandemic, largely to avoid infection risk in the registration process, but with follow-on positive impacts on the long-term development of CRVS systems.

Now, after more than 1 year's experience of coping with the COVID-19 pandemic it is essential for governments to reflect on and evaluate the effectiveness of the strategies they took to deal with the longer term impact on CRVS systems. A follow-up survey to the one reported on here is now timely. This survey could document the longer term impact of COVID-19 on CRVS operations on the completeness and timeliness of notification and registration, the success and effectiveness of moves to digitise services, the impact on ascertainment of cause of death, and the impact on data management and compilation of vital statistics, and the quality evaluation of these statistics [18]. Countries must also take this opportunity to utilise the lessons learnt from these evaluations to catalyse broader CRVS development, and some areas where that may be possible are outlined in this paper. Finally, there is an important role in this 
process for the international organizations that work on CRVS development particularly from the United Nations convened Global CRVS Group. These organizations can provide guidance to countries in how to conduct such evaluations, and compile international data and share outcomes across countries. This will allow for the development of international strategies for sustainable CRVS strengthening which can also be tailored to individual countries.

\section{CONFLICTS OF INTEREST}

The authors declare they have no conflicts of interest.

\section{AUTHORS' CONTRIBUTION}

All authors conceptualised the project and contributed to the analysis design. MK led the analysis and write up. All authors edited and approved the final draft.

\section{REFERENCES}

[1] United Nations. Statistics Division. Principles and Recommendations for a Vital Statistics System. 2014. Available from: https://unstats.un.org/unsd/demographic/standmeth/ principles/M19Rev3en.pdf [cited February 18, 2021].

[2] Jackson D, Wenz K, Muniz M, Abouzahr C, Schmider A, Braschi MW, et al. Civil registration and vital statistics in health systems. Bull World Health Organ 2018;96;861-3.

[3] Rao C. Elements of a strategic approach for strengthening national mortality statistics programmes. BMJ Global Health 2019;4;e001810.

[4] Zylke JW, Bauchner H. Mortality and morbidity: the measure of a pandemic. JAMA 2020;324;458-9.

[5] Vestergaard LS, Mølbak K. Timely monitoring of total mortality associated with COVID-19: informing public health and the public. Euro Surveill 2020;25;2001591.

[6] United Nations. UN Legal Identity Agenda: impact of COVID-19. 2020. Available from: https://unstats.un.org/legal-identityagenda/covid-19 [cited February 18, 2021].
[7] United Nations Legal Identity Agenda Task Force. Maintaining Civil Registration and Vital Statistics during the COVID-19 pandemic. New York, USA: UNLIATF; 2020.

[8] United Nations Development Programme. Guidance to UNDP Country Offices on the privacy, data protection and broader human rights dimensions of using digital technologies to combat Covid-19. 2020. Available from: https://unstats.un.org/legalidentity-agenda/documents/unlia-guidance-to-undpOffices.pdf [cited February 18, 2021].

[9] World Health Organization (WHO). International guidelines for certification and classification (coding) of COVID-19 as cause of death. 2020. Available from: https://www.who.int/ classifications/icd/Guidelines_Cause_of_Death_COVID-19.pdf [cited February 18, 2021].

[10] Vital Strategies and World Health Organization. Revealing the toll of COVID-19: a technical package for rapid mortality surveillance and epidemic response. New York: Vital Strategies; 2020.

[11] Rao C. Medical certification of cause of death for COVID 19. Bull World Health Organ 2020;98;298-A.

[12] World Health Organization (WHO). WHO methods and data sources for country-level causes of death 2000-2019. Geneva: Department of Data and Analytics (DNA), Division of Data, Analytics and Delivery for Impact (DDI), World Health Organization; 2020.

[13] Global surveillance for COVID-19 caused by human infection with COVID-19 virus: interim guidance, 20 March 2020. Geneva: World Health Organization; 2020. Available from: https://apps. who.int/iris/handle/10665/331506 [cited March 20, 2021].

[14] Cardoso K, Shveda K. Measuring Africa's data gap: the cost of not counting the dead. London, UK: BBC News; 2021.

[15] Armario C. A pandemic atlas: Peru's death toll leaves a grieving nation. New York, USA: AP News; 2020.

[16] Cabrera JML, Kurmanaev A. Ecuador's death toll during outbreak is among the worst in the world. New York: New York Times; 2020.

[17] The Hindu. Release data on registered deaths to understand mortality impact of COVID-19. New Delhi: The Hindu; 2020.

[18] The Pacific Community. Impact of the COVID-19 pandemic on operations of national Civil Registration and Vital Statistics (CRVS) systems. Noumea, New Caledonia: Pacific Community, Statistics for Development Division; 2021. 